\title{
ViII.
}

\section{Il viaggio di Berkeley in Sicilia ed i suoi rapporti con un filosofo poeta.}

\author{
per \\ Mario-Manlio Rossi (Firenze).
}

Mentre oggi la filosofia di Berkeley viene studiata largamente, la vita di lui non ha negli ultimi tempi dato luogo a studi o scoperte interessanti. Come giá notóil Lorenz¹), la biografia ${ }^{2}$ ) e le due edizioni critiche delle oper $\mathrm{e}^{3}$ ) dovute al Fraser, per quanto complete, sono in molti luoghi difettose e non sono state fino ad oggi migliorate, se non da alcuni saggi ${ }^{4}$ ) del Lorenz su questioni speciali. Come continuazione di questo lavoro di documentazione, riprodurró qui due lettere di B. che rimangono ancora sconosciute alla maggior parte degli studiosi, ed insieme porteró alcune notizie sulla vita di B. nel 1718 e sul suo corrispondente, Tommaso Campailla ${ }^{5}$ ' di Modica

1) Th. Lorenz, Weitere Beiträge zur Lebensgeschichte G. Berk. (Arch. f. Gesch. d. Phil., XVIII, p. 551). Vedi anche „Mind“, 1902, p. 249 .

2) A. C. Fraser, Life and letters of G. B. Oxford 1871.-

3) The works of G. B. Voll. III. Oxford 1871, e: The works of G. B. incl. his posth. works. Voll. IV. Oxford 1901.

4) Arch. f. Gesch. der Phil., XIII, p. 541; XIV, p. 293; XVIII p. 159 ; XVIII, p. 551 .

5) Membro corrispondente di varie accademie italiane, cavaliere, ecc. vissuto dal 1668 al 1740 .

6) La stessa prefazione fu poi riprodotta in tutte le edizioni successive. La prima edizione é del 1728 (la dedica peró é datata 20 febbraio 1729) - Messina, stamperia Chiaramonte e Provenzano. Poi: 1737, Roma (Palermo?), A. Rossi; 1744 (non 1757 come vuole il Di Giovanni) Milano, G. Cairoli. Finalmente, l'edizione delle opere complete in due voll. in folio (1783 - Siracusa, F. M. Pulejo), preceduta da un saggio biografico di $\mathrm{S}$. Sinesio. 
(Sicilia), poiché nessuno dei biografi di B. há finora notati questi rapporti. Né il Fraser né il Lorenz mostrano di conoscerli e soltanto alcuni scrittori italiani poco importanti e poco noti, che di B. conoscono soltanto il nome, accennano ad essi.

Queste due lettere vennero probabilmente comunicate dallo stesso Camp. al suo concittadino Jacopo De Mazara ed Echebelz, che le inserí nella prefazione apologetica della prima edizione del l'opera principale del Camp ${ }^{6}$ ). In tempi piú recenti solo il filosofo siciliano Vincenzo Di Giovanni ha preso in considerazione questi documenti ${ }^{7}$ ), studiandoli peró soltanto in rapporto al Camp. per concluderne, come giá aveva fatto il De Mazara ${ }^{8}$ ), che B. teneva in gran conto il pensiero filosofico del Camp. ${ }^{9}$ ). Chiunque osservi con attenzione i seguenti documenti attribuirá senz'altro tale opinione ad una esagerazione campanilistica del reale-valore di questi.

(1)- Messanae, Februarii 25, 1718 Clarissime vir. „Ex itinere per universam insulam instituto iam tandem, favente Numine, reversus, animum jucundissima memoria Siculorum hospitum, atque amicorum, praesertim quos ingenio, atque eruditione praestantes inviserim subinde reficio. Porró inter illos quanti te faciam, Vir doctissime, facilius mente concipi, quam verbis exprimi potest. Id unum me mali habet ,quod ex audito tuo colloquio diutiús frui per itineris festinationem non licuerit. Clarissimos ingenii tui fructus, quos mihi impartire dignatus sis, quamprimúm Londinum pervenero, aequis illiusmodi rerum Aestimatoribus distribuendos curabo. Si quid interim aliud occurrat, quod ad Societatem Regiam Londinensem transmitti cupias, id, modo mittatur ad D. D. Porten, Hoaré, et Allen Anglos, negotii causa Messanae commorantes, ad me, ubicumque tandem sim, perveniet; Porro Neutoni nostri Naturalis Philosophiae principia Mathematica, si quando in Patriam sospes rediero, ad te transmittenda dabo, vel si qua alia ratione commodis

7) St. della filosofia in Sicilia - Palermo, 1873, p. 282, 288: vedi anche Sinesio, op. cit., p. 36. - Dell' esistenza di questi documenti e del loro interesse per la vifa di B. soltanto G. Papini si mostrò al corrente, benche non accenni ad essi nel suo interessantissimo studio sul $\mathrm{B}$. (,Il Rinnovamento", 1908, p. 235 - riprodotto in "Ventiquattro cervelli", Firenze 1912, p. 71).

8) op.cit., ch. b-2: ,Solanente qui sotto aggiungeróla testimonianza che ne (dell'importanza filosofica del Camp.) fa il sig. Giorgio Berkeley, ora graduato in Irlanda."

9) „Tenne commercio di lettere con molti - fra i quali il Berkeley - e fu onorato vivente di bella fama presso le dotté accademie di Londra, ..." (Di Giovanni, op. cit., p. 282.) 
tuis inservire possim, reperies me, si minús potentem, promptum tamen, omnique ossequio, Humillimum servum G. Berkeley.

(2)- Londini Kalendis Julii 1723. Clarissime vir. Post longam quinque fermé annorum peregrinationem, variosque casus, et discrimina, nunc demum in Angliam redux, nihil antiquius habeo, quám fidem meam, tibi quondam obbligatam. Deus bone! Ab illo tempore quot clades, quot rerum mutatione, tam apud vos, quám apud nos! Sed mittamus: haec tristia. Libros tuos, prout in mandatis habui, Viro erudito é societate Regia tradidi, qui, cum solertiam, et ingenium tuum pro meritis. extimet, túm id plurimum miratur, tantum Scientiae lumen in extremo Siciliae angulo tám diú delituisse. Telescopium quod attinet Catoptricum, é metallo confectum, id quidem olim agressus est Neutonum; verúm res ex voto non successit: nam impossibile erat, nitidum chalybis splendorem usque é conservare, ut stellarum imagines distincté exhiberet; proinde huiusmodi Telescopia, nec in usu sunt, nec unquam fuere; nec, praeter unicum illud, quod Author, experimenti causâ fabri cavit, ullum factum esse unquam, vel fando accepi. Hodie certé apud nostrates non reperiuntur. Caeterúm librum clarissimi istius Philosophi iuxta, ac Mathematici, quem spondebam missurum, ad te mitto, quem tamquam sincerae amicitiae pignus accipias, quaeso. Tu interim, Vir clarissime, promovere rem literariam, pergas; artesque bonas, et scientias in ea Insula serere, et propagare, ubi felicissimae terrae Indoles frugibus, et ingeniis apta ab omni aevo aequé fuit. Scito, me tibi semper futurum Addictissimum, et humillimum servum G. Berkeley.

Non si puó dubitare dell'importanza di questi documenti, data la scarsitá e l'incertezza delle notizie sul viaggio in Sicilia di B. Il Fraser ${ }^{10}$ ), richiamandosi ad un accenno d'una lettera di B. ${ }^{11}$ ) ed a brevi passi di due opere storicoletterarie ${ }^{12}$ ), ci fa sapere soltanto che B. nel $1718 \mathrm{fu}$ in Sicilia e precisamente a Messina, e. viaggiando a piedi per l'isola, raccolse osservazioni di storia naturale, che peró andarono perdute insieme al giornale di viaggio nel ritorno a Napoli.

Possiamo ora preci:ar meglio queste notizie. Tanto la lettera (1)

10) Life, p. 84 .

11) Del 1745, nella quale B. afferma di aver sentito un terremoto a Messina nel 1718.

12) Memoirs of the Court of Augustus, by Dr. Blackwell (trad. franc. Feutry) - Paris, Cellot, 1781. - An Essay on the Genius and Writings of Pope, by Warton = London, Dodsley, 1782, p. $200 \mathrm{seg}$., vol. II: "He not only made the usual tour, but - even travelled on foot through Sicily." 
quanto un passo della prefazione del De Mazara ${ }^{13}$ ) attestano anzitutto che B. parló di persona con il Camp. E poiché tutti i biografi affermano concordemente che ilfilosofo siciliano non si allontanó mai, in tutta la sua vita, da Modica ${ }^{14}$ ), ne dobliamo concludere che B. nel suo viaggio si spinse fino a Modica. Verosimilmente il viaggio fu abbastanza breve: al 22 ottobre 1717 B. era ancora a Napoli ${ }^{15}$ ) ed il 25 febbrajo 1718 era giá di ritorno a Messina ${ }^{16}$ ). Si deve credere che egli partisse da Napoli negli ultimi mesi del 1717, perché il suo soggiorno in Sicilia sarebbe stato troppo breve se si fosse limitato, come sembra intenda il Fraser ${ }^{17}$ ), all'anno 1718. Non avrebbe potuto cioé esser giá di ritorno il 25 febbraio dopo aver viaggiato, in parte anche a piedi, per universam insulam, raccogliendo svariate osservazioni e note di viaggio. Si noti a questo proposito che il Fraser ${ }^{18}$ ) parla di ,materials for a natural history of the island“, mentre il Warton ${ }^{19}$ ) fa capire chiaramente che B. in Sicilia osservò soprattutto gli avanzi di antichi monumenti in rapporto all'architettura. Infatti Modica ${ }^{20}$ ) é vicina all'antica Siracusa.

In tutti i modi rimane un poco dubbia l'indicazione della lett. (1): „p er universa m insulam“. B. non poté certo compiere tutto il viaggio a piedi; ed in tutti i modi, nonostante la suddetta indicazione, B. non può aver avuto il tempo di visitare la parte sudoccidentale dell' isola. Nulla di certo possiamo dire su questo soggetto: bisognerebbe cercare qualche lettera od altri documenti relativi ai rapporti di $\mathrm{B}$. con i tre commercianti inglesi di Messina (non menzionati da

13) Il Sinesio anche afferma (saggio biogr. cit.) che il B., come molti altri, si reco presso di lui, "tratto dalla sua fama".

14) Era tanto pauroso della morte da rinchiudersi in uno stanzino con le pareti imbottite, senza uscirne fer tutta la stagione fredda (cfr. Di Giovianni, op. cit., p. 279).

15) Abbiamo una sua lettera datata da Napoli del 22 ottobre 1717 (Fraser, · p. 82).

16) Quando scrisse la lett. (1) aveva proprio finito il viaggio in Sicilia e si disponeva ad abbandonare l'isola: ,iam tandem - reversus".

17) "He appears to have been also in. Sicily in that year" (p. 84).

18) loc. cit.

19) "B. gained the patronage and friendship of Lord Burlington - by his profound and perfect skill in architecture: an art which he had very particularly and accurately studied in Italy - Sicily, and drew up an account of that very classical ground ..." (op. cit.).

20) Oggi, una cittadina di ca. 50000 abitanti. 
alcun biografo), se si volessero piú precise notizie sulle varie questioni che restano insolute circa il viaggio in Sicilia.

La conoscenza fra i due filosofi fu dunque transitoria, certo non tale da farci pensare che B. stimasse Camp. proprio come pensatore e si intrattenesse con lui per ammirazione della sua filosofia ${ }^{21}$ ). E'ben naturale che B., incontrando in quel luogo per lui cosí remoto, „,in extremo Siciliae angulo", uno studioso, volesse informarsi della sua linea di pensiero: ma senza per questo divenirne un fervente ammiratore, come vorrebbero i critici siciliani giá nominati. Le lettere citate non sono in realtá documento particolare di stima o di ammirazione, per cosí dire, specificamentefilosofica: si tratta piú che altro di espressioni di riconoscenza per un ospite gentile, per una buona conoscenza di viaggio. E'certo che se il De Mazara avesse trovate altre lettere di B. a Camp. si sarebbe curato di pubblicarle, 0 almeno di accennare ad esse: mi sembra invece certo che il commercio epistolare fra i due si sia limitato a queste, poiché non troviamo notizia di altre in nessuna opera, e il De Mazara, che appare, col Sinesio ${ }^{2 \varepsilon}$ ), cosí perfettamente informato della vita del Camp., arrebbe notato, nel caso, che erano andati perduti, o comunque gli mancavano, ulteriori documenti, se avesse saputo di un vero scambio di lettere.

I rapporti fra B. e Camp. si limitarono dunque presumibilmente al primo colloquio, all' invio di due o tre libri, alla presentazione di lavori del Camp. ad un membro ${ }^{23}$ ) della Royal Society. Si noti anzi che non sappiamo l'esito di questo interessamento del B., che probabilmente non fu troppo caldo, poiché né gli studiosi del B. né quelli del Camp. ne trovarono altra traccia. Rapporti dunque di cortese camaraderie sold:nto, con invio di libri e di notizie scientifiche, e nemmeno duraturi. Non poteva del resto avvenire diversamente, se si ponga mente alle profonde divergenze speculative fra i due, che appariranno evidenti dalle poche notizie che seguono

2i) Anzi il Sinesio annovera anche B. fra quelli che „tratti dalla sua fama - non dipartivansi contenti senza conoscerlo e visitarlo . .."

2a) Il De Mazara era evidentemente suo concittadino ed intimo, il Sinesio poi då moltissime notizie nel saggio prefisso, come si disse, all' edizione del 1783 .

23) Che fu probabilmente quello stesso Arbuthnot che comunico alla R. S. le osservazioni di B. sul. Vesurio. 
e che accludo per inquadrare debitamente questo episodio, poiché 1a figura del Camp. ritengo sia sconosciuta, o quasi, alla maggior parte degli studiosi, anche italiani.

Dovremo peró prima accennare ad alcune deduzioni che possiamo trarre circa la vita di B. dalla lett. (2). Questa prova che solo dopo piủ di due anni dal suo ritorno in patria, eforse dopo ulteriori sollecitazioni del Camp., B. si curó di dargli conto delle commissini affidategli, affermando che solo allora era ritornato, mentre sappiamo che era in Inghilterra giá dalla fine del $1720^{24} \lambda$. Si ricordi che nel $1723 \mathrm{~B}$. dimorava abitualmente a Dublino, perché lettore di ebraico nel Trinity College; si recava a Londra solo per brevi soggiomi. Probabilmente questa volta si trovava a Londra per pratiche relative alla ereditá di Ester Vanhomrigh, il testamento della quale fu aperto appunto il 6 giugno $1723^{25}$ ). In ogni caso, é interessante poter cosí stabilire che nel mese successivo alla morte di Vanessa, B. fu senza dubbio a Londra, e forse per un po' di tempo: si ricordi l'episodio un poco misterioso della distruzione da parte di B. della corrispondenza di Vanessa e Swift.

Nelle opere piú recenti e piú largamente note agli studiosi non si trovano accenni al nome ed all' opera del Camp., se si eccettuano poche righe del Bouillier, che d'altronde non ne eita il nome ${ }^{23}$ ), e varî passi del Di Giovanni ${ }^{27}$ ), poco noto peró fuori d'Italia. Solo in tempi relativamente lontani il Camp. diede luogo a qualche monografia ed a sparsi accenni in opere di erudizione minuziosa 0 di storia regionale ${ }^{28}$ ). La fama di lui non varcó in fondo i confini

${ }^{24)}$ Fraser, p. 87.

25) Fraser, p. 99.

28) „des vers italiens cités par Gerdil, d'un poéte et philosophe de son temps qu' il ne nomme pas" (Bouillier, Hist. de la phil. cart., vol. II ${ }^{0}$, p. 523).

27) Op. cit., ๆ. 278, segg. - Vedi inoltre accenni in „Della filosofia moderna in Sicilia; Filosofia e lett. sic.; ecc. Per molte notizie biografiche e bibliografiche sono inde bitato a queste opere accuratissime.

${ }^{28}$ ) Ne riporto alcune per chi voglia avere ulteriori informazioni: Salvo Di Pietraganzili, St. della lett. in Sicilia, 1. I0, p. 9. - La Lumia, St. sic., vol. IV0, p. 553. - Sciná, Prosp. della lett. sic., e Inzenga, Comp. eiusdem - Villabianca, Saggio fil. sul poema, in: Apocalisse ecc. - ed. 1784 - Villareale, Saggi estetici e critici - Ortolani, Biogr. degli it. 
della Sicilia: le sue opere furono infatti poche e tutte di scarsissimo valore filosofico ${ }^{29}$ ); solo il poema filosofico „L'Adamo“ ebbe, ma al suo tempo soltanto, qualche fortuna e viene citato nelle storie letterarie, inesattamente, come imitazione del „Paradise Lost“. Si tratta in realt à di un' opera farraginosa e noiosa, in XX canti d'ottave, nella quale nulla troviamo, né dal lato artistico né da quello filosofico, che giustifichi l'appellativo, dovuto al Muratori, di Lucrezio Cristiano ${ }^{30}$ ). Con questo il grande storico si riferiva forse alla propensione del Camp. per la filosofia atomistica, soprattutto nella esposizione del Gassendi ${ }^{31}$ ). Ma questa male si accorda, se non per esteriori rapporti, col cartesianesimo prevalente nel pensiero del Camp. Questi indica esplicitamente, con lodi sperticate, gli autori preferiti ${ }^{32}$ ); accenna alla Royal Society, ma non cita né Locke né altri pensatori della scuola inglese: altra prova questa della differenza fondamentale di vedute fra lui e B., di cui tutti poi ricorderanno la profonda avversione sí alle dottrine atomistiche che a quelle cartesiane ${ }^{33}$ ). Di piú, il Camp. si mostra sempre ferventissimo

ill., vol. II0, p. 87 - Mongitore, Bibl. sic. - Amico; Diz. top. sic. Trieste-Bovio, in : Opusc. del Calogerá, vol.X - Melchiorre da S.Antonino, Oraz. in lode ecc. - Gallo, ne ,I'Imparziale“ di Firenze, anno I0 - 20. Poi saggi critici e biografici di Navarro, Ventura, Guastella, Grana.

${ }^{29}$ ) L' Adamo ovvero Il Mondo Creato; L'Apocalisse dell' apostolo S. Paolo; Considerazioni sulla fisica di Newton (presentato all'Acc. franc $_{\text {) }}$; Problemi; Discorso sul moto interno degli animali; Discorso sulla fermentazione; ecc.

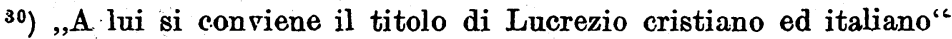
(lett. àl Prescimone, editore ed epitomatore del poema) - „Avendo noi in quell' autore un nuovo Lucrezio" (lett. al Ceva).

31) Nel canto I parla dell'atomi e del vuoto come principi delle cose; vedi anche canto V; str. 21-22, 51. - Tra gli altri opuscoli, ne scrisse uno „Della fermentazione", argomento specificamente lucreziano (vedi anche i canti IV, XV, ecc.).

${ }^{32}$ ) Nel canto V soprattutto. - Notiamo fra gli altri Boyle, Bayle, i filosofi e naturalisti italiani del suo tempo: come si vede, é impossibile par lare d'un suo pensiero personale.

${ }^{33}$ ) Soprattutto nel Commonplace Book. - Si ricordi la cura con cui B.nel III Dialogo si differenzia da Malebranche. Notiamo che Camp. afferma in una lettera al Muratori (1730): ,mi sono distaccato da Cartesio nel luogo del senso comune ch' ei pone nella glandola pineale"c - B. derise la theoria stessa nel „Guardian" (,The Pineal Gland of a Free-thinker", in: Works - ed. 1871, III, p. 154). 
Berkeley in Sicilia ed i suoi rapporti con un filosofo poeta. 163

ammiratore del Newton $\left.{ }^{i}\right)$, come si puó anche rilevare dalle lettere succitate, mentre $B$. combatté questi in varie opere e per varie dottrinerespingendo quella teoria della gravitazione di cui Camp. si mostra invece entusiasta ${ }^{35}$ ). I rapporti fra i due filosofi non poterono davvero essere troppo intimi, né fondarsi sull' ammirazione dell' uno per il pensiero dell' altro, se Camp. si rivolse proprio a B. per aver notizie e libri di Newton. Con ogni probabilitá, il filosofo siciliano non conosceva né le opere né il pensiero di B. e d'altra parte questi non si curó di parlargliene: in questo caso $B$. avrebbe inviato i suop libri al Camp. E nemmeno Camp. diede le sue opere a B. perché lui stesso le leggesse, ma soltanto glie le affidó perché le consegnasse a chi si sarebbe interessato di idee per le quali B. personalmente non aveva alcuna simpatia: Abbiamo poi una prova definitiva del fatto che lo stesso Camp. non dava troppa importanza alla conoscenza del B. Quando avvenne questa, L'Adamo non era ancora compiuto: il De Mazara afferma infatti che le opere di Camp. presentate alla Royal Society per il tramite di B. consistevano nel discorso „Der moto degli animali" ed in alcune copie dei primi canti ${ }^{36}$ ). Doobbiamo quindi ritenere che l'opera, allora, non fosse compiuta ${ }^{37}$ ). Ora, se Camp. avesse conosciuto, approvandolo o no, il pensiero del B., avrebbe potuto accennare a lui ne $L$ 'Adamo, dove, come si disse, accenna a quasi tutti i filosofi contemporanei che conosceva. Invece il pensiero del B. si era formato su altri autori da quelli prediletti del Camp. e si era svolto per vie diversissime.

Il Camp. dedica gran parte del poema a tutte le questioni: naturali allora di moda: dal punto di vista filosofico, credo che le sole cose importanti siano i giudizi sui filosofi contemporanei e l' espo-

34) Ricordiamo i vari passi delle due lettere, le „Considerazioni sopra la fisica d' I. Newton", ecc. ecc.

$\left.{ }^{35}\right) \mathrm{Nel}$ „De Motu“ e nei „Princ. of Hum. Kn.", CIII. Vedi inoltre il Cmpl. Book - ed. 1901, p. 85 ecc.; , ,The Analist"; ,A Defence of freethinking in mathematics", contro le teorie matematiche di Newton.

36) loc. cit.

37) Il Di Giovanni par la veramente di una ,stampa di Catania del 1709" (op. cit., p. 284), ma non pare fosse completa, né ho potuto. trovarne altra traccia. Dalla prefazione del Prescimone e da quella del De Mazara risulta che, in ogni caso, tutto il poema non àpparve. che nel 1728. Infatti l'edizione del 1737 é indicata nel titolo come ,questa. seconda edizione". 
sizione poetica delle varie teorie cartesiane $\left.{ }^{38}\right)$. Nella stessa epoca il Genest (1717) e dopo di lui il Polignac e B. Stay, conterraneo del Camp. tentarono la stessa impresa. esposizione il Camp. si richiama chiaramente a $\mathrm{S}$. Agostino ${ }^{39}$ ): ne fu infatti assiduo lettore e fu probabilmente attraverso ad esso che cominció ad interessarsi della filosofia cartesiana di cui fu primo seguace in Sicilia.

Basteranno questi pochi dati per comprendere l' importanza puramente regionale del Camp. e la superficialitá dei suoi rapporti con il B. Per una piú larga conoscenza del pensiero di lui, rimandiamo alle stesse sue opere ed agli studi del Di Giovanni.

38) Rispettivamente, canto $V$ e canto $I$.

39) „Pensando d'ingannarmi al. certo io penso" (canto I). Beuché nella lettera al Muratori (cit. Di Giovanni, op. cit., p. 292) dove, dopo aver detto che "tutto il nerbo" della sua filosofia è cartesiano, elenca poi le differenze, non citi fra queste la sua accezione del cogito ergo sum, mi sembra chiara l' influenza dell' Ipponense, con vantaggio, a mio credere, del fondamento gnoseologico, se si intenda questo nel suo reale valore, di reductio ad absurdum dello scetticismo. 